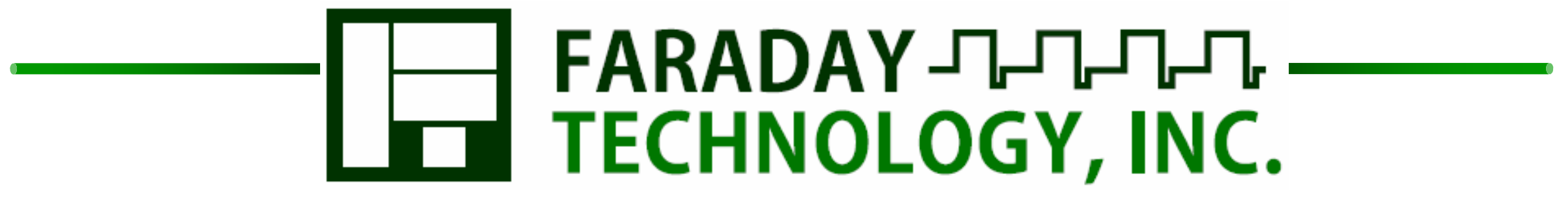

\title{
Low Cost Corrosion and Oxidation Resistance Coatings for Improved System Reliability
}

\author{
Jing $X \mathbf{u}^{1}$ \\ Timothy D. Hall ${ }^{1}$ \\ Maria Inman' \\ E. Jennings Taylor ${ }^{1}$ \\ Stephen T. Snyder ${ }^{1}$ \\ Ying Zhang ${ }^{2}$ \\ May 15, 2018
}




\section{Challenge}

\section{Problem}

Current coating processes are costly or incompatible with coating complex shape components and currently can not maintain performance required at the next generation operating temperatures

\section{Turbine Disks / Blades}

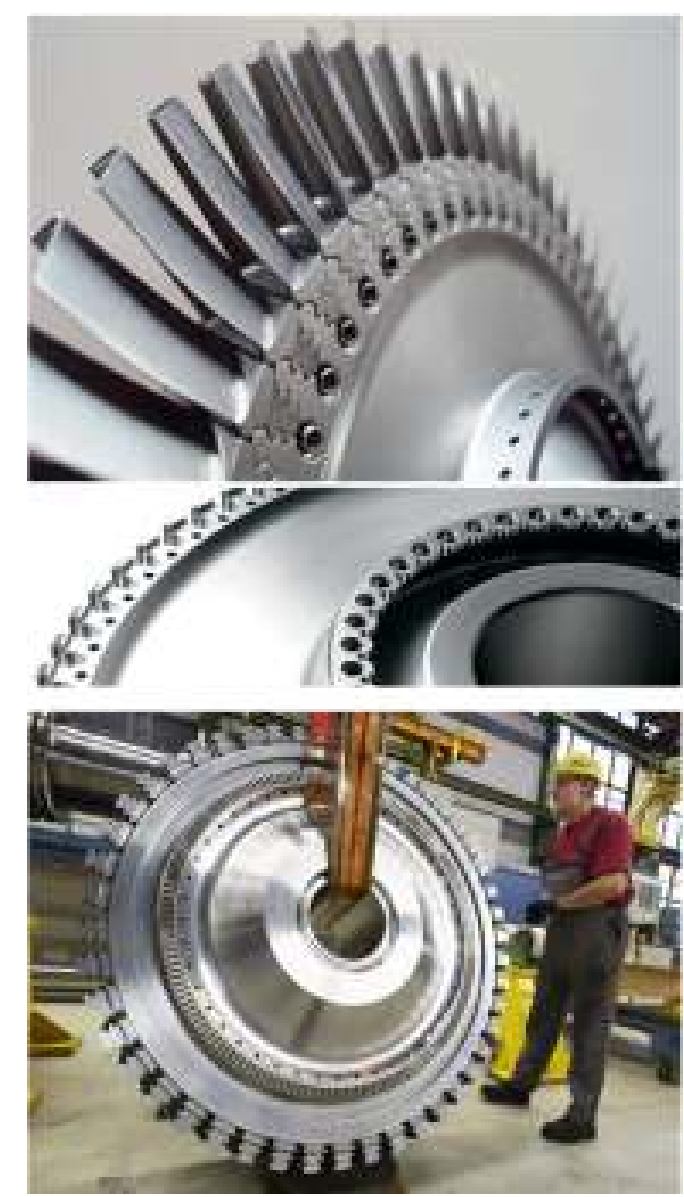

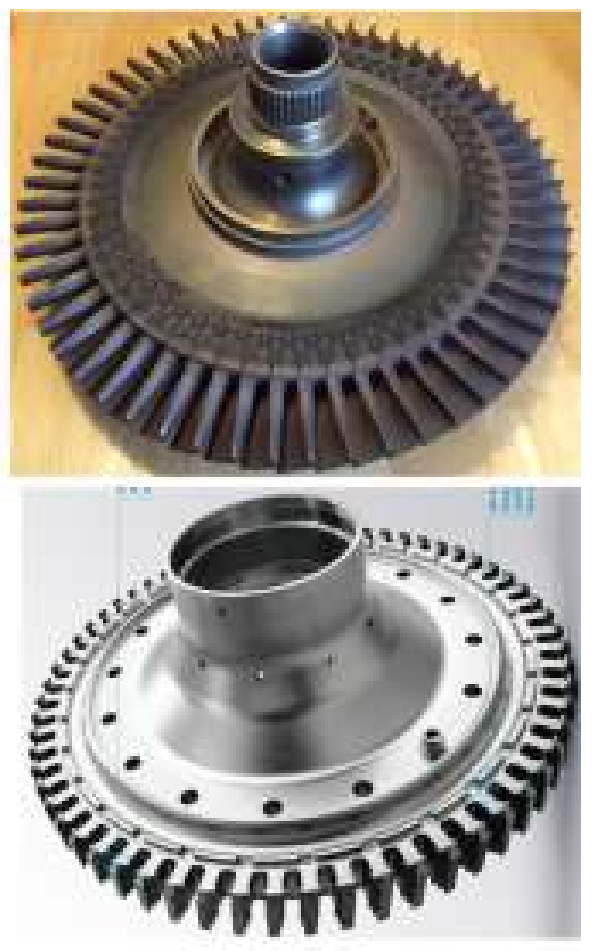




\section{Goal/Objective}

- Develop a scalable cost effective process to produce coatings that can enhance high temperature reliability and corrosion/oxidation resistance.

- Evaluate the potential of designing new state of the art coatings, based on our scalable approach, to further improve resistance to operating temperatures in excess of $760^{\circ} \mathrm{C}$.
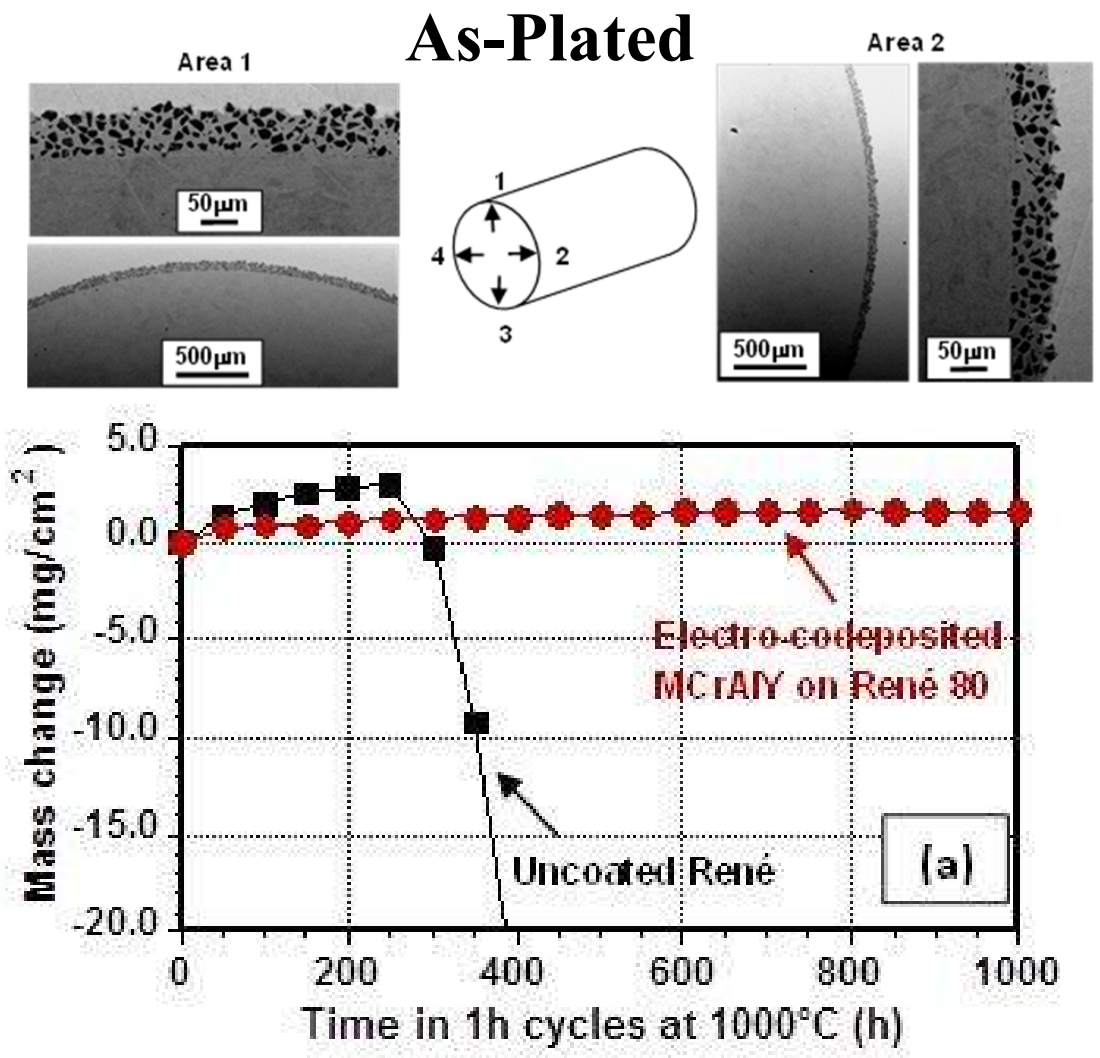

FARADAY ЛПЛП TECHNOLOGY, INC. 


\section{Technical Approach}

- Design a custom bench-scale system to electrochemical codeposition NiCo with CrAlY powder

- Heat treat and evaluate the coating for potential high temperature corrosion and oxidation resistance

- Estimate the scalability and potential cost savings

- Design an alpha scale apparatus

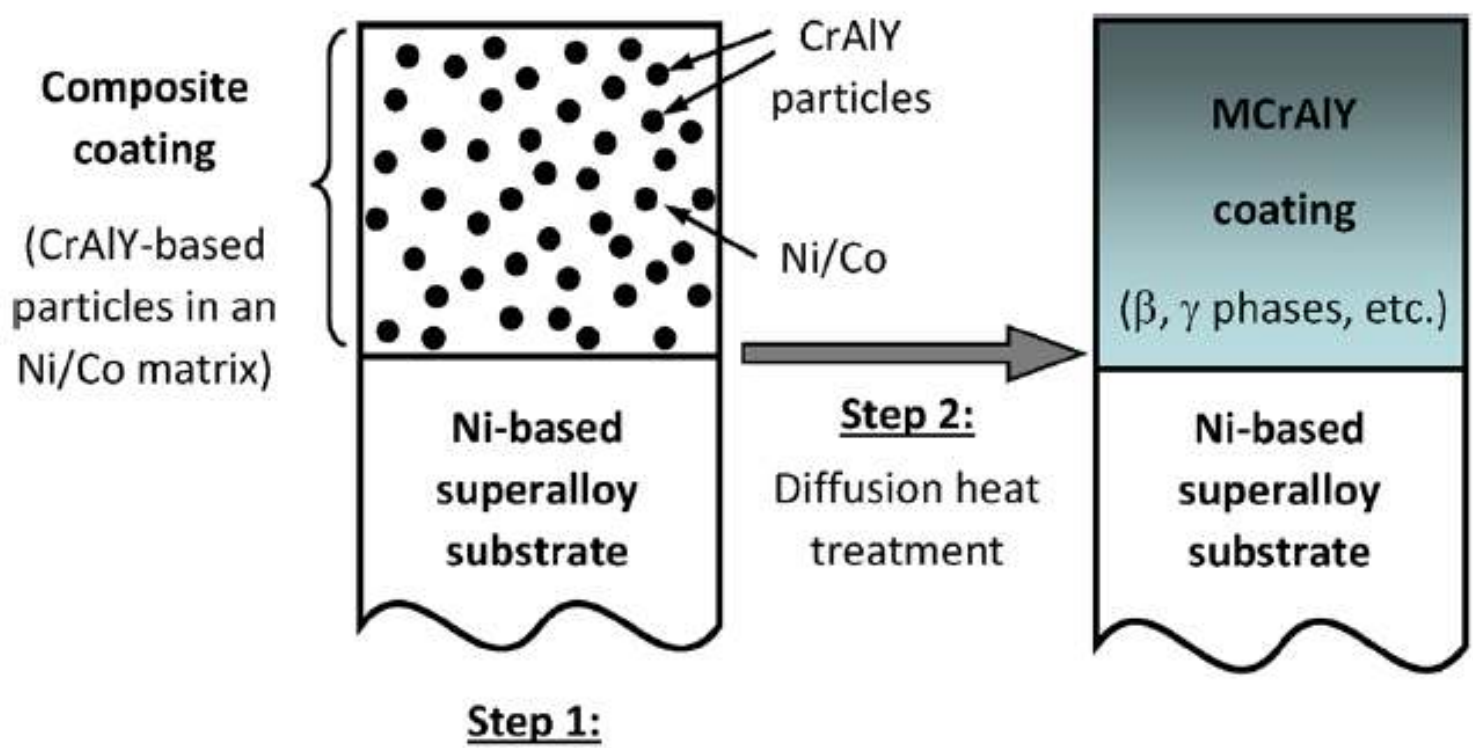

Electro-codeposition of 


\section{Material and Testing}

- Turbine Disk Material, Shape, and Composition

Ideally ME3, LSHR, or Alloy10

- Used Inconel (IN718) due to availability

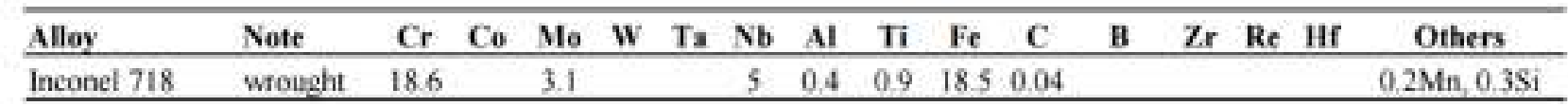

- Coating Deposition and Characterization

- Analyzed the coating via XRF and SEM-EDX to measure composition and cross-sectional uniformity in the as-plated and post heat treatment orientations

- Coating Consolidation and Mixing

- After plating high temperature anneal

- Critical Tests

- High Temperature Corrosion

- Low Cycle Fatigue (LCF)

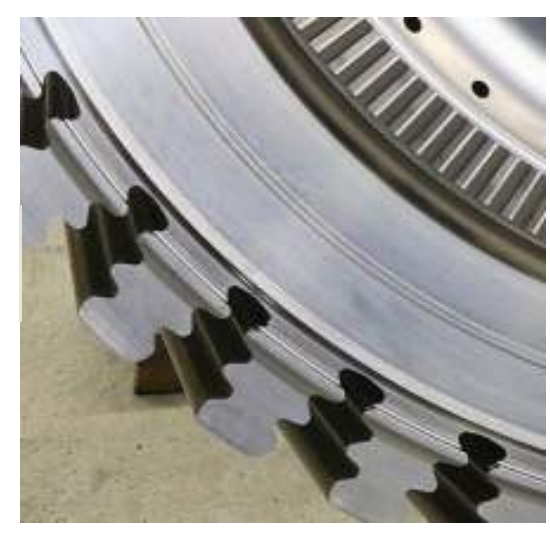

- Plated bar and demonstrated coating

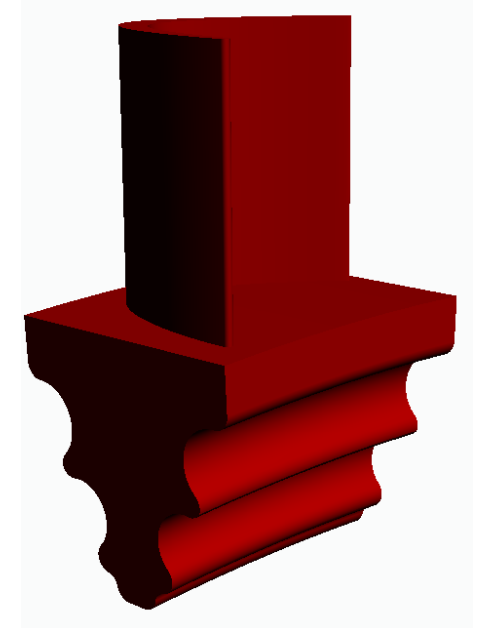




\section{Experimental setup and Materials}

- Substrate

- IN718 Tubes

- Base electrolyte components

- $\mathrm{NiSO}_{4}, \mathrm{CoSO}_{4} 5 \mu \mathrm{m}$ CrAlY powder

- Designed and built reactor
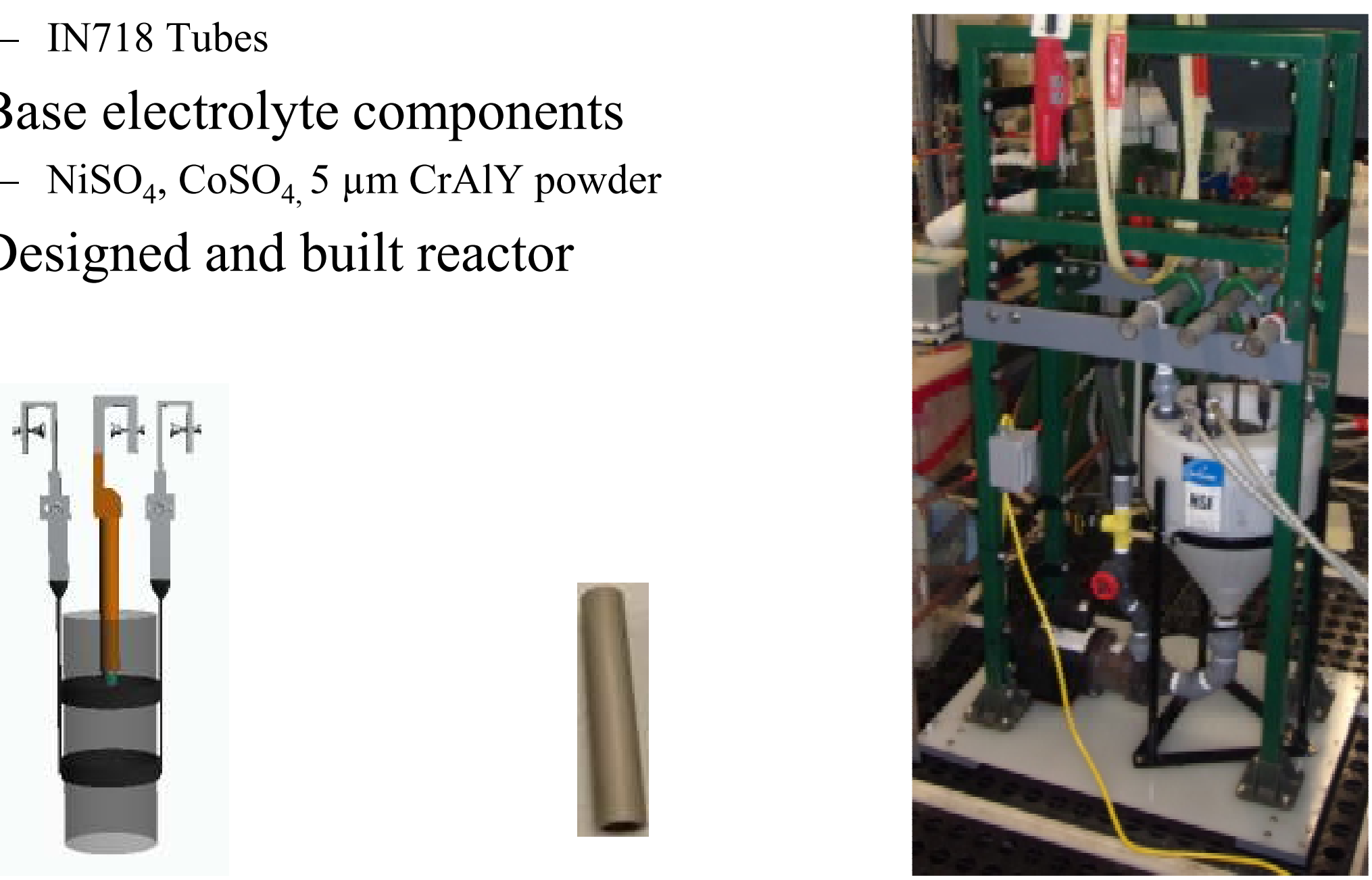

FARADAY ЛПЛת TECHNOLOGY, INC. 


\section{Materials (CrAIY \& Cr powders)}

- $5 \mu \mathrm{m}[\mathrm{Cr}, 29-31 \% \mathrm{Al}, 1.4-2 \% \mathrm{Y}, 0.4 \% \max \mathrm{O}, 0.3 \% \max \mathrm{Fe}, 0.1 \% \max$ $\mathrm{Si}, 0.06 \% \max \mathrm{C}, 0.05 \% \max \mathrm{N}]$ powder from Sandvik

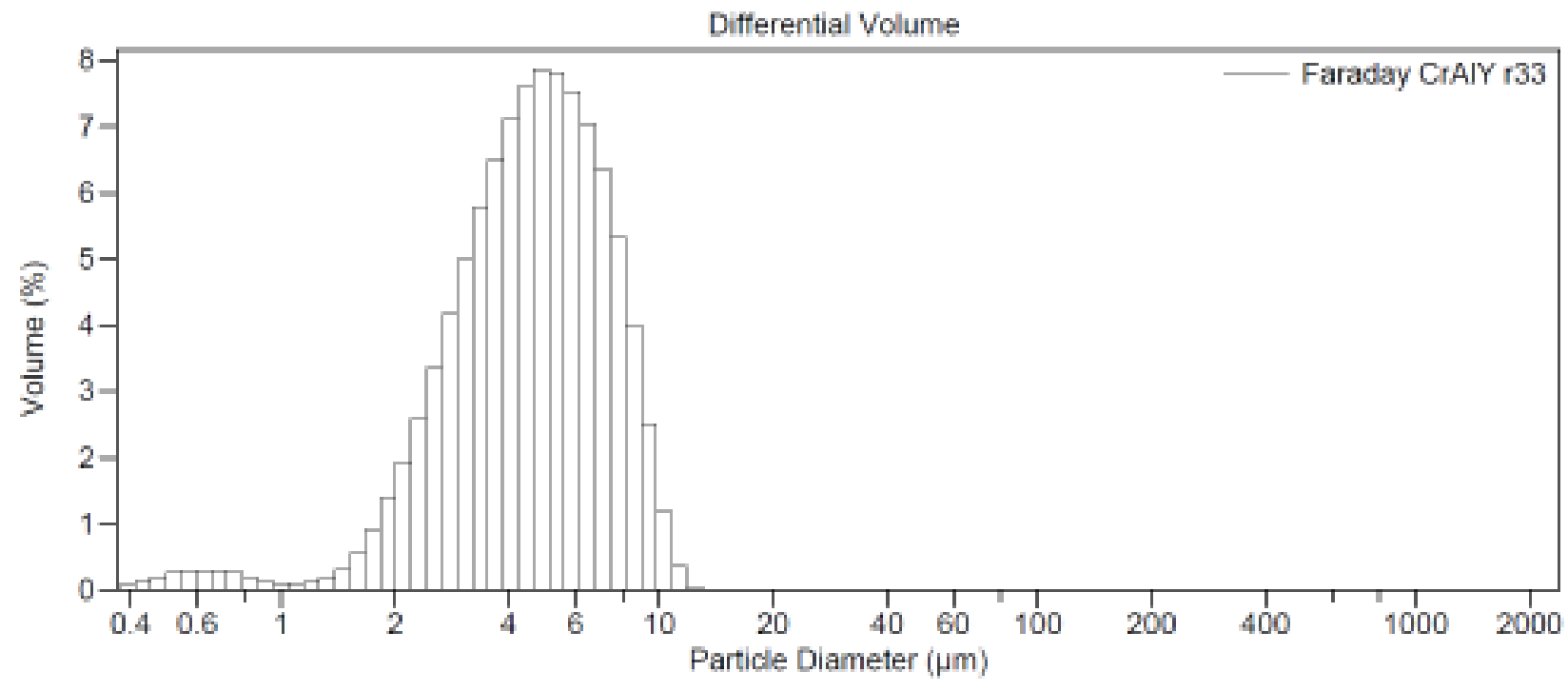

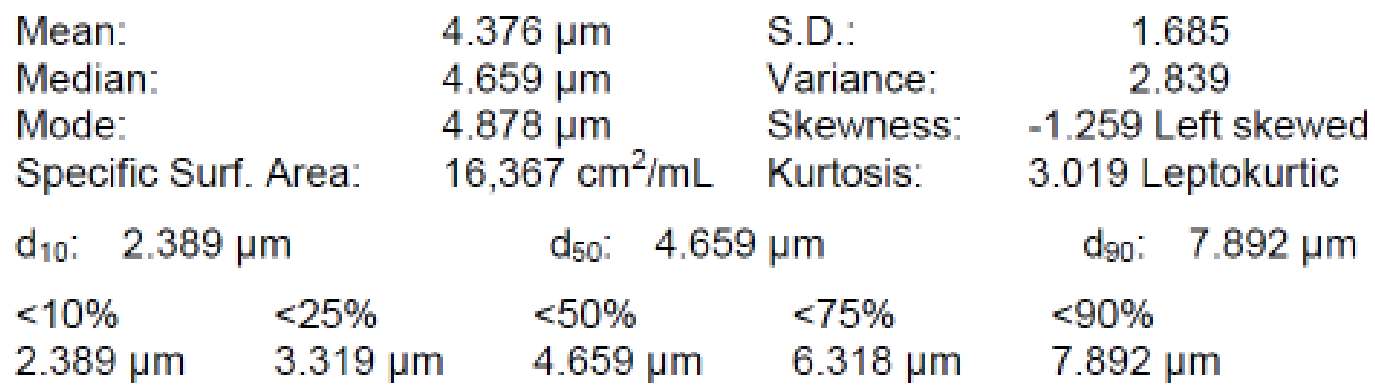




\section{Coating Compositional Development}

- Substrate and Materials

- Inconel 718 and Nimonic 105 disc samples $(\sim 16 \mathrm{~mm}$ in diameter and $2 \mathrm{~mm}$ thick)

- CrAlY powder

- $\mathrm{Ni} / \mathrm{Co}$ electrolyte containing $100 \mathrm{~g} / \mathrm{L}$ powder

- Heat treatment: $6 \mathrm{~h}$ at $1000^{\circ} \mathrm{C}$
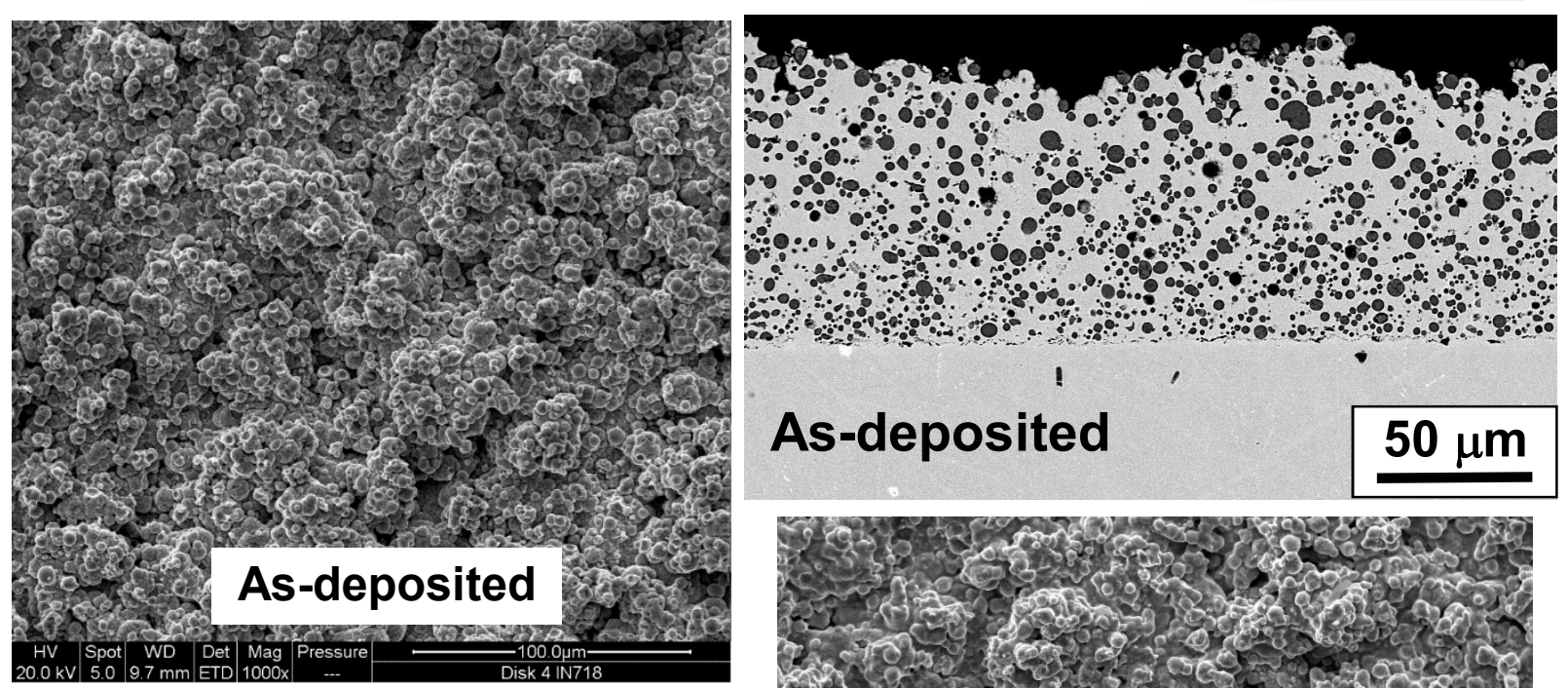

- Analysis

- The NiCoCrAlY coating appears very uniform

- EDS analysis indicates the coatings contain approximately 8-10 wt.\% $\mathrm{Al}$ and 12-15 wt.\% Cr after heat treatment.

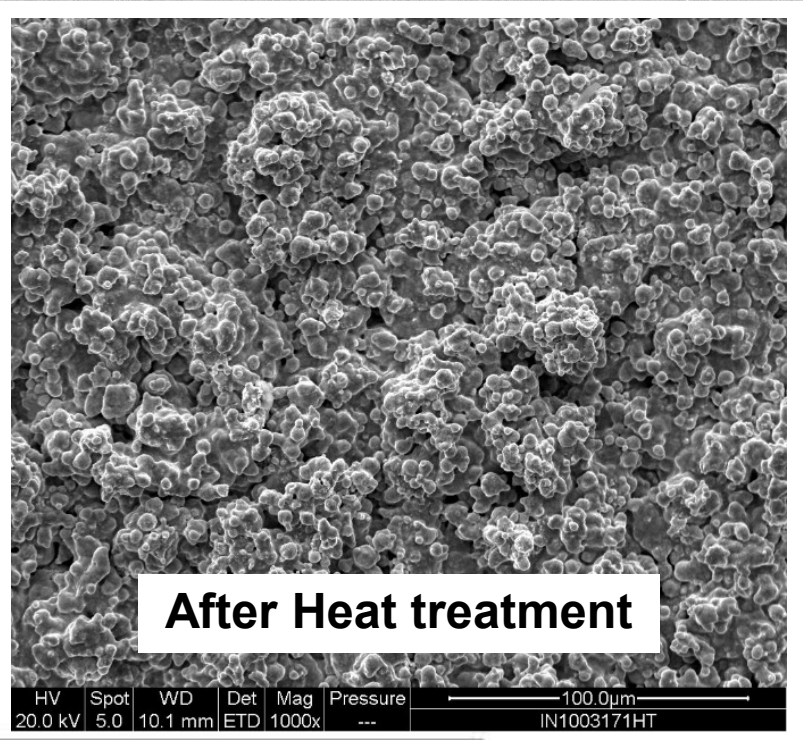

\begin{tabular}{llccccccccccccccc}
\hline Alloy & Note & Cr & Co & Mo & W & Ta & Nb & Al & Ti & Fe & C & B & Zr & Re & Hf & Others \\
\hline Inconel 718 & wrought & 18.6 & & 3.1 & & 5 & 0.4 & 0.9 & 18.5 & 0.04 & & & $0.2 \mathrm{Mn}, 0.3 \mathrm{Si}$ \\
\hline \hline Nimonic 105 & wrought & 14.5 & 20 & 5 & & & & 1.2 & 4.5 & & 0.2 & & \\
\hline
\end{tabular}




\section{Experiment - Tank Trials (CrAlY powder)}

- Plated up to $12 \mathrm{wt} \% \mathrm{CrAlY}$ powder within the $\mathrm{NiCo}(78 \% \mathrm{Ni} / 10 \% \mathrm{Co})$ matrix - $\mathrm{pH}$ control is crucial for a uniform and adherent coatings
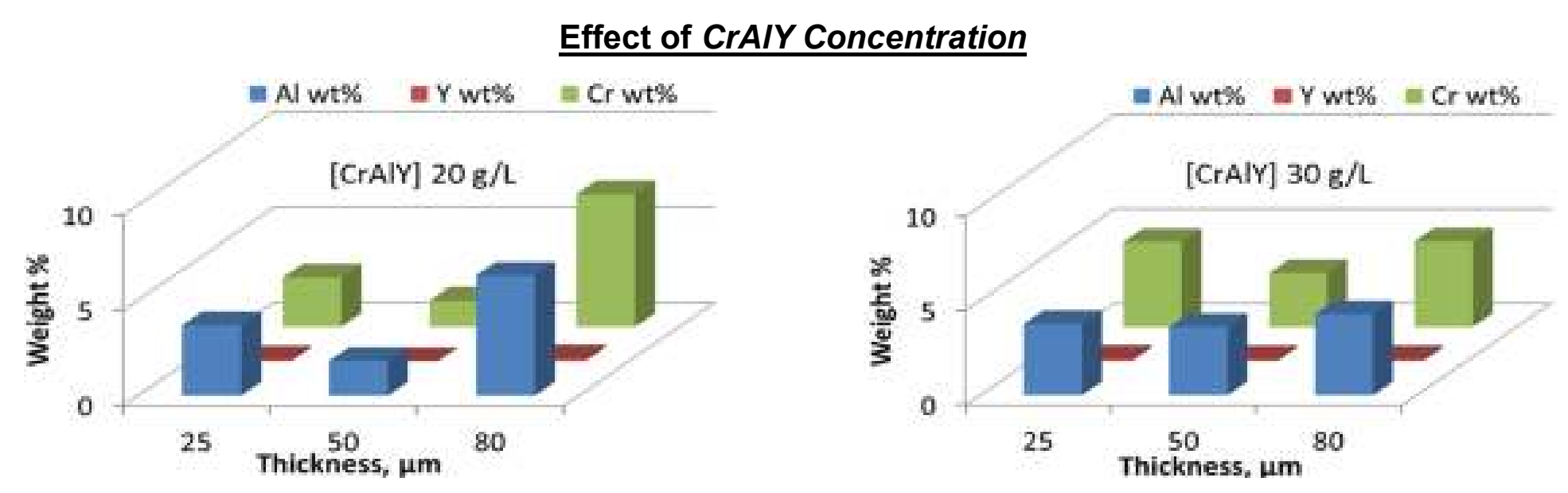

Effect of $p H$
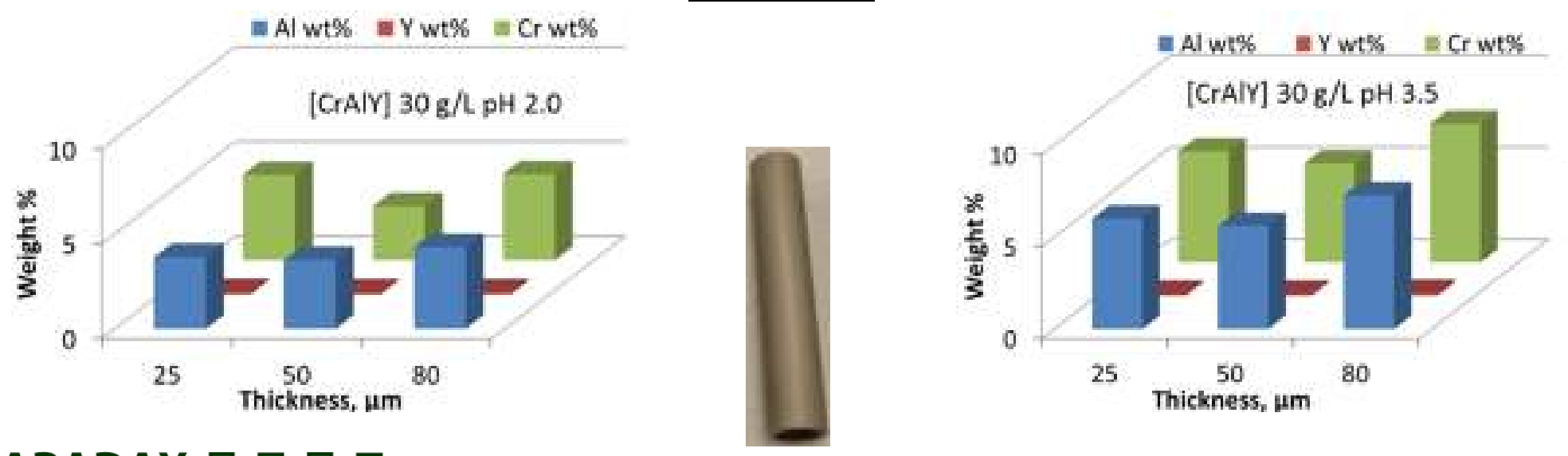

FARADAY תחת TECHNOLOGY, INC. 


\section{Cross Section (CrAlY powder)}

\section{Effect of $\mathrm{pH}$ on the coating before annealing}

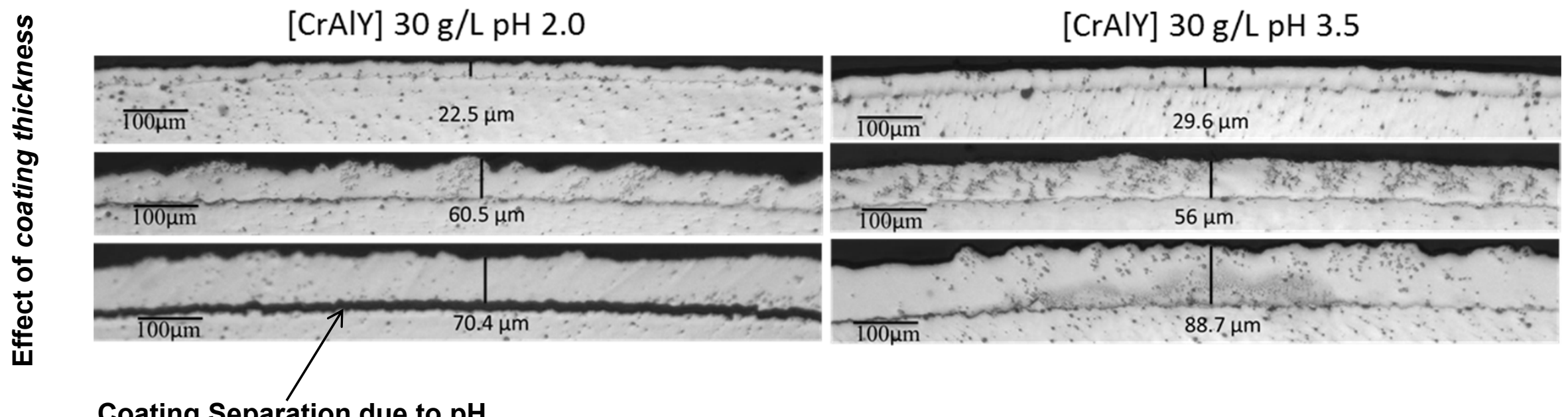

Coating Separation due to $\mathrm{pH}$

[CrAlY] $30 \mathrm{~g} / \mathrm{L} \mathrm{pH} 2.0$

[CrAlY] $30 \mathrm{~g} / \mathrm{L} \mathrm{pH} 3.5$

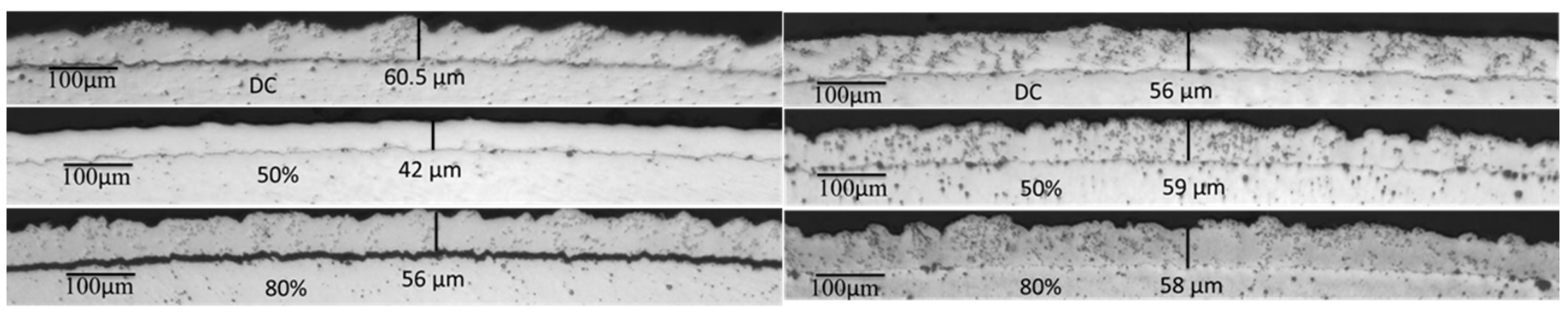

FARADAY ЛПЛЛ TECHNOLOGY, INC. 


\section{Annealing Development}

- Annealing Treatment (Using Tube Furnace at TTU)

$-8 \mathrm{~h}$ at $760^{\circ} \mathrm{C}$ in Ar performed on LCF bars

$-6 \mathrm{~h}$ at 800,850 and $1000^{\circ} \mathrm{C}$ in vacuum

- Found a minimum of $850^{\circ} \mathrm{C}$ required to fully anneal

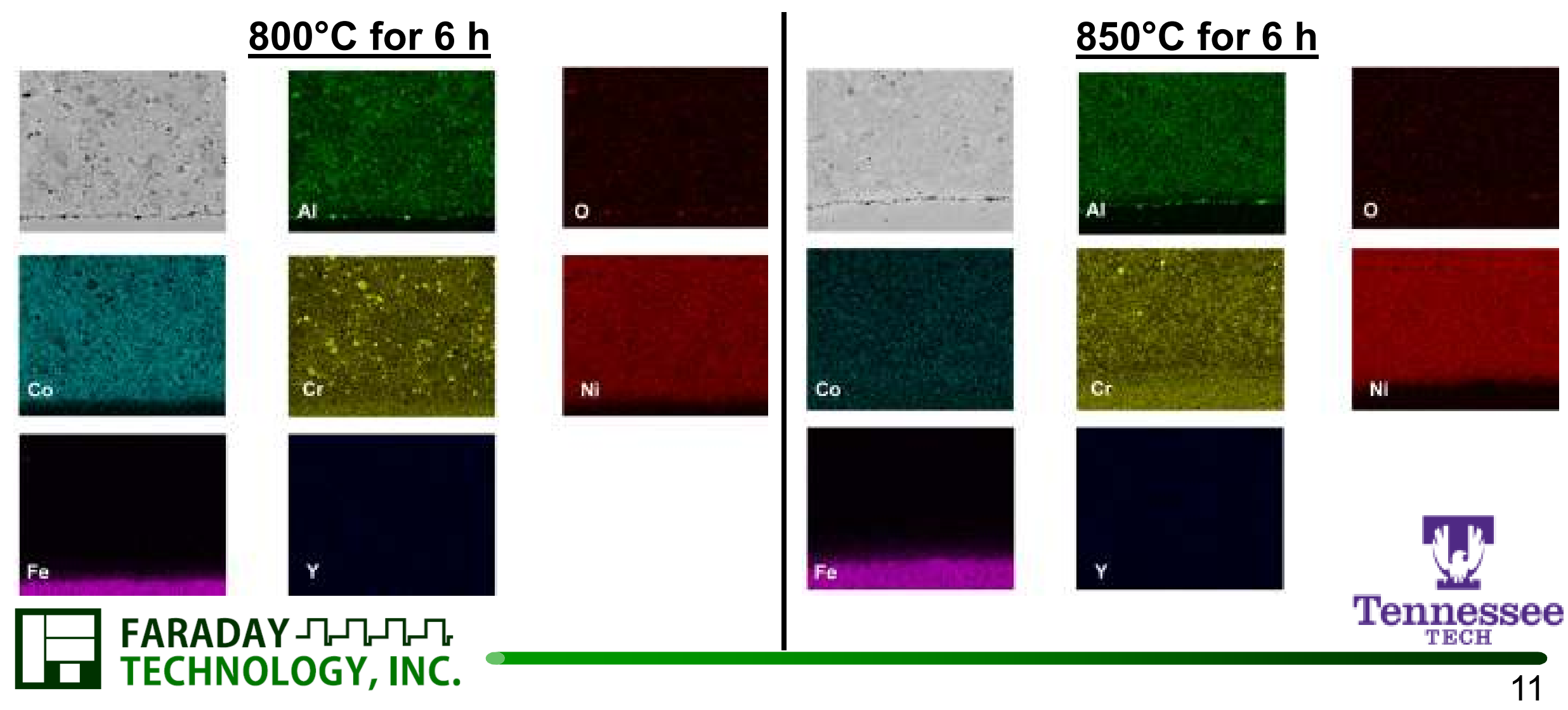




\section{Corrosion Testing}

- Oxidation trials at $800^{\circ} \mathrm{C}$ in air (after $3100-\mathrm{h}$ )

- All specimens showed very low mass gains after oxidation $\left(0.1-0.32 \mathrm{mg} / \mathrm{cm}^{2}\right.$

IN 718 Uncoated
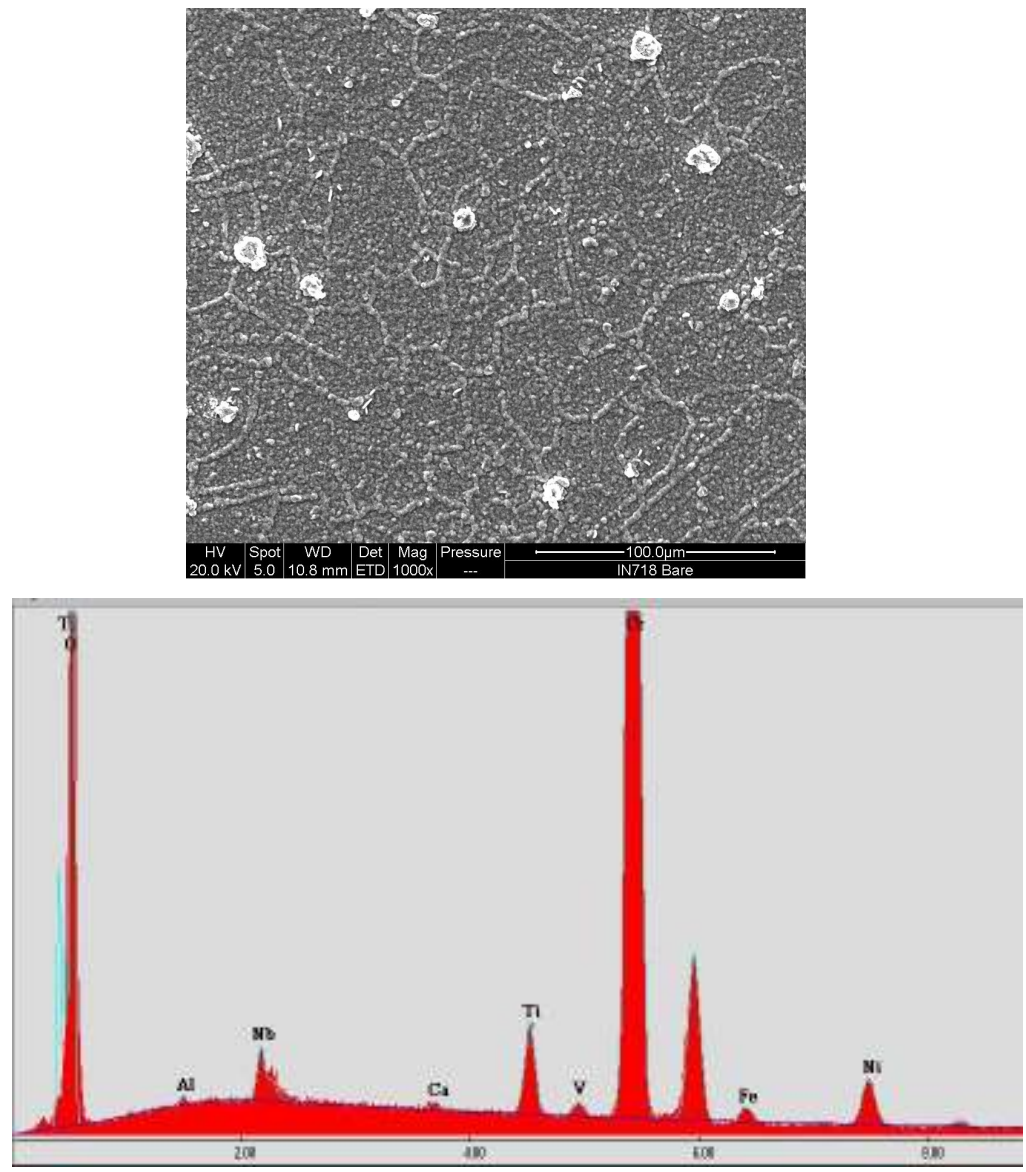

FARADAY ЛПЛЛ TECHNOLOGY, INC.
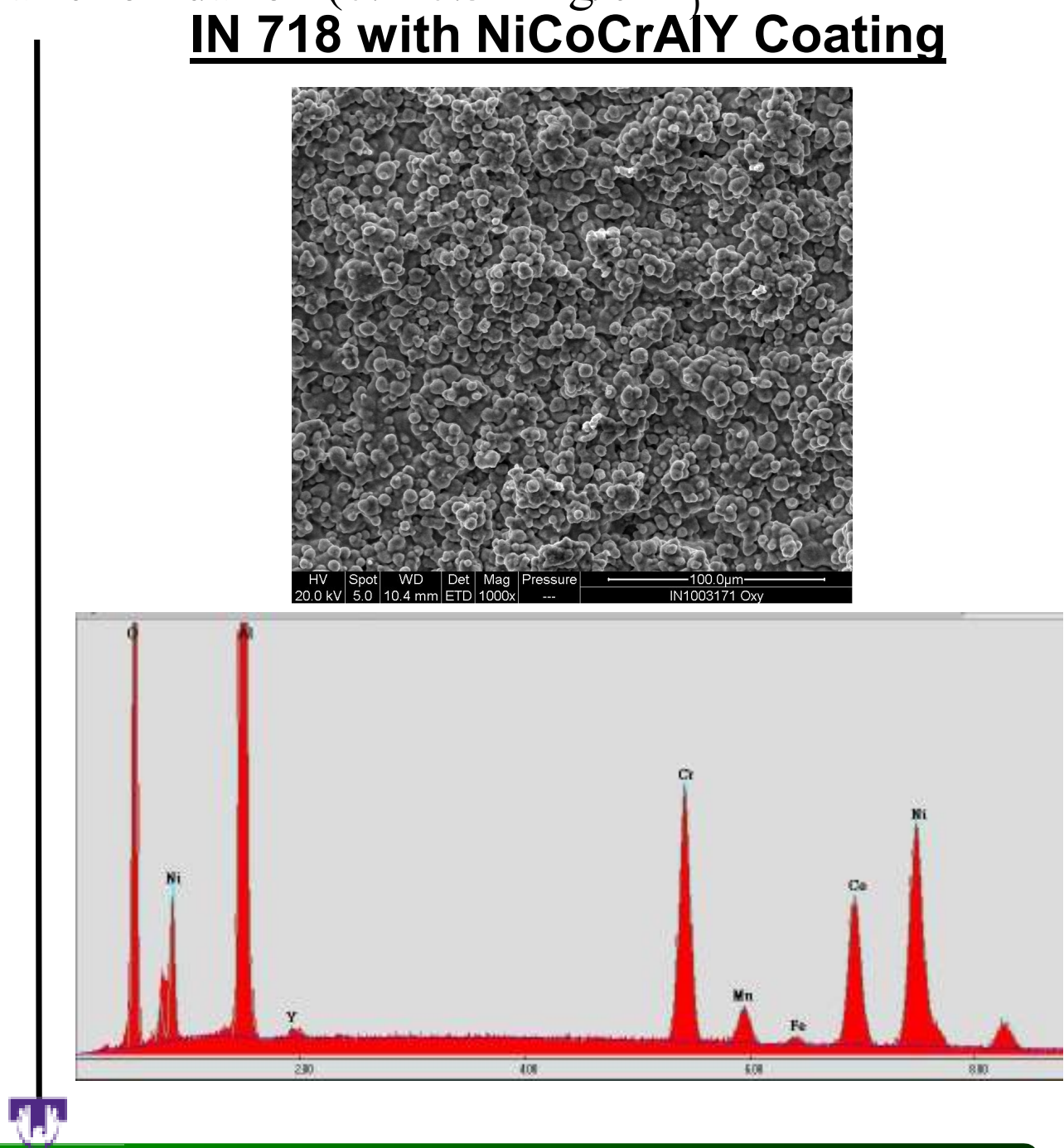


\section{Corrosion Testing}

- 50h at $760^{\circ} \mathrm{C}$ in static air ; $55 \% \mathrm{Na}_{2} \mathrm{SO}_{4}-45 \% \mathrm{MgSO}_{4}$, Salt deposit: $2.6-2.7 \mathrm{mg} / \mathrm{cm}^{2}$

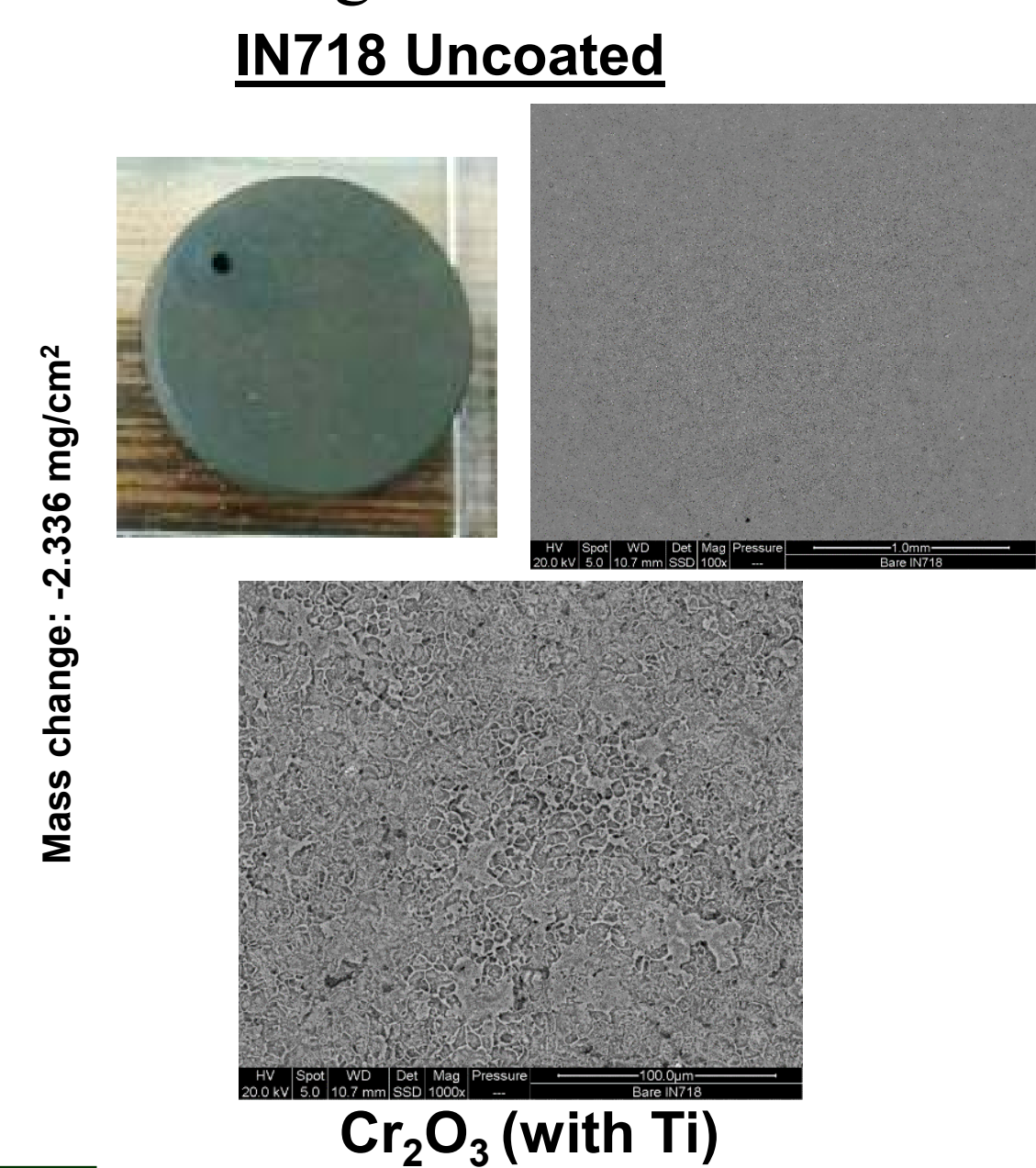

FARADAY ЛПЛЛ TECHNOLOGY, INC.

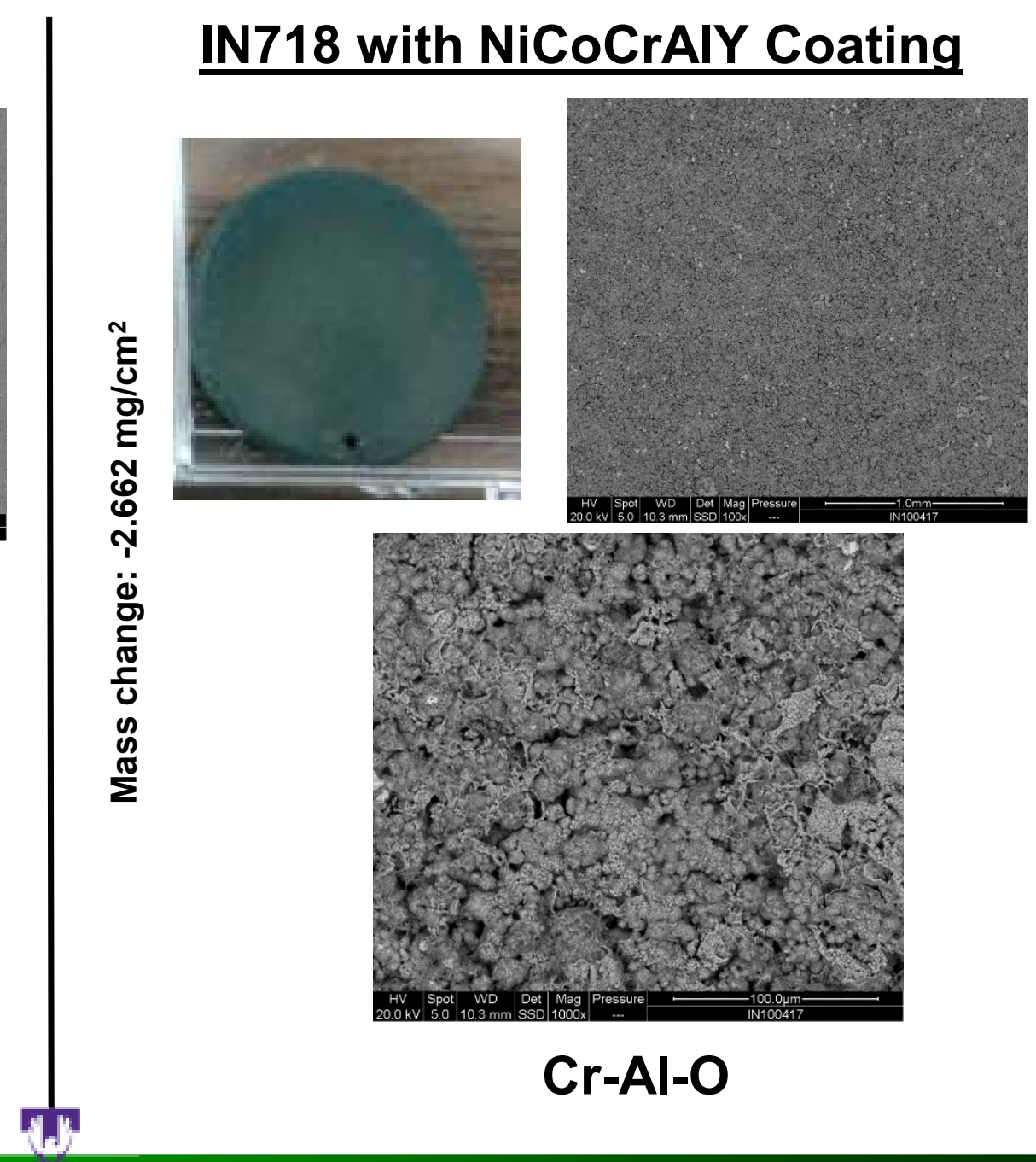

Tennessee 


\section{Corrosion Testing}

- 50h at $760^{\circ} \mathrm{C}$ in static air ; $55 \% \mathrm{Na}_{2} \mathrm{SO}_{4}-45 \% \mathrm{MgSO}_{4}$, Salt deposit: $2.6-2.7 \mathrm{mg} / \mathrm{cm}^{2}$
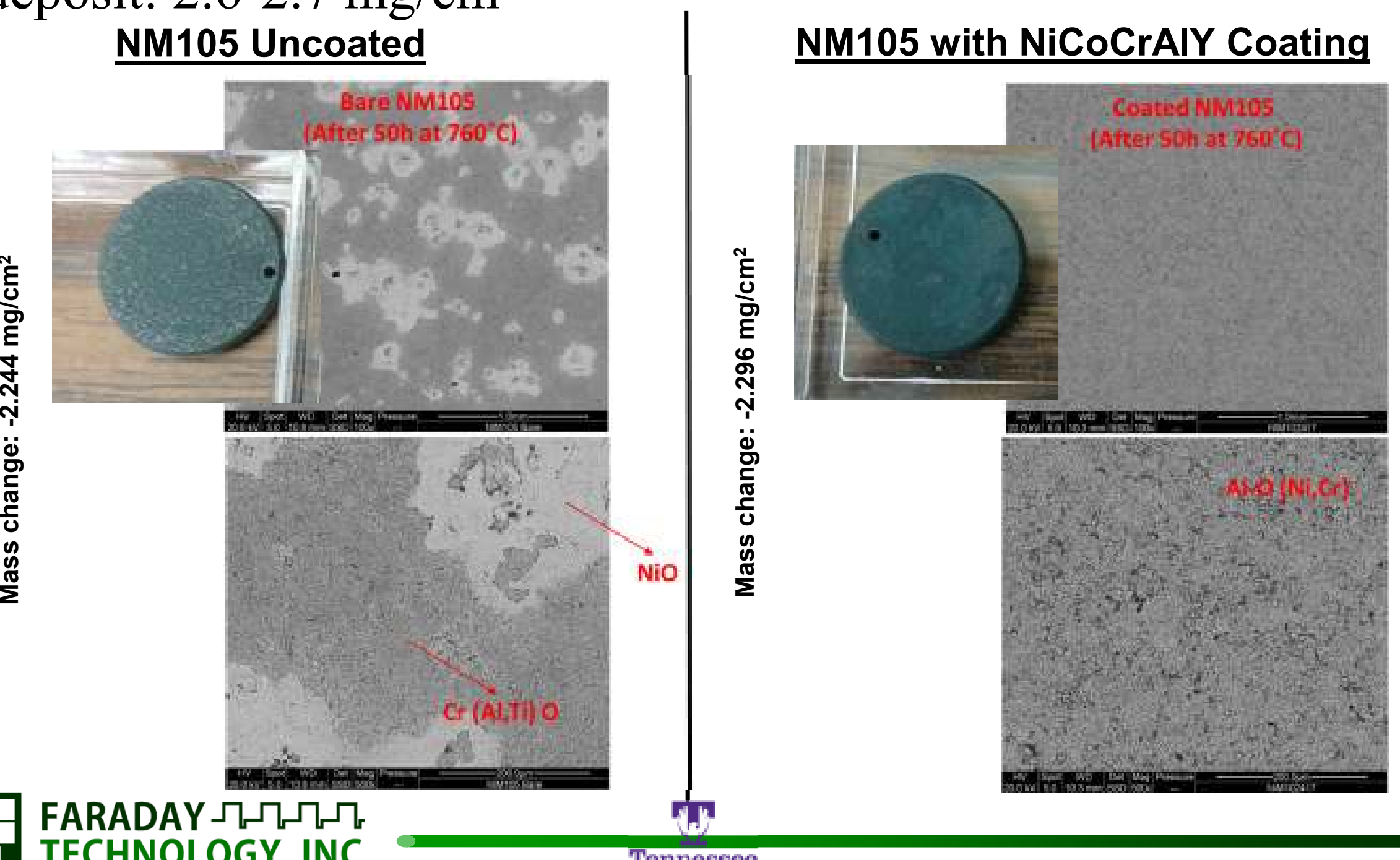

TECHNOLOGY, INC. 


\section{LCF Bar plating}

\section{As Plated}
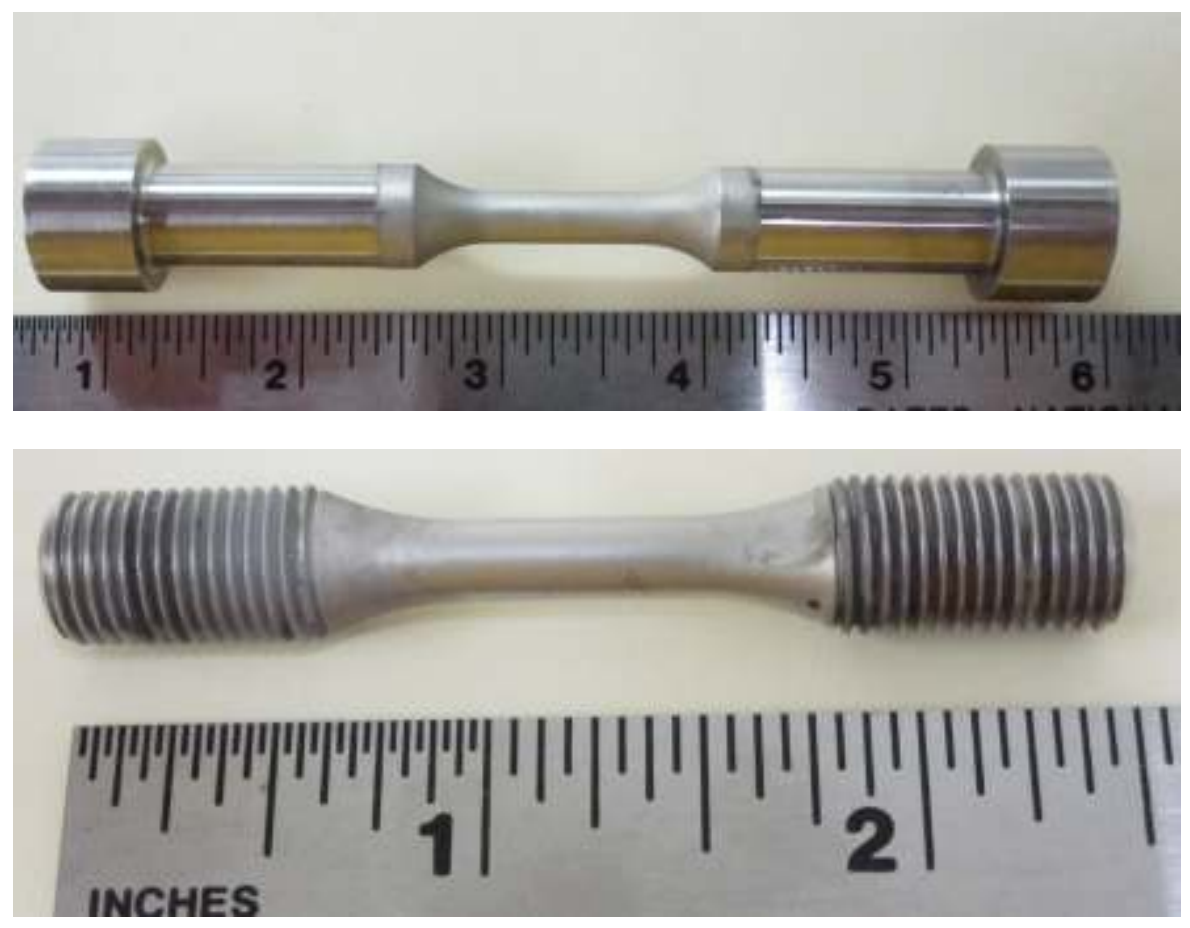

Post Annealed @ 760 $\mathrm{C}$.
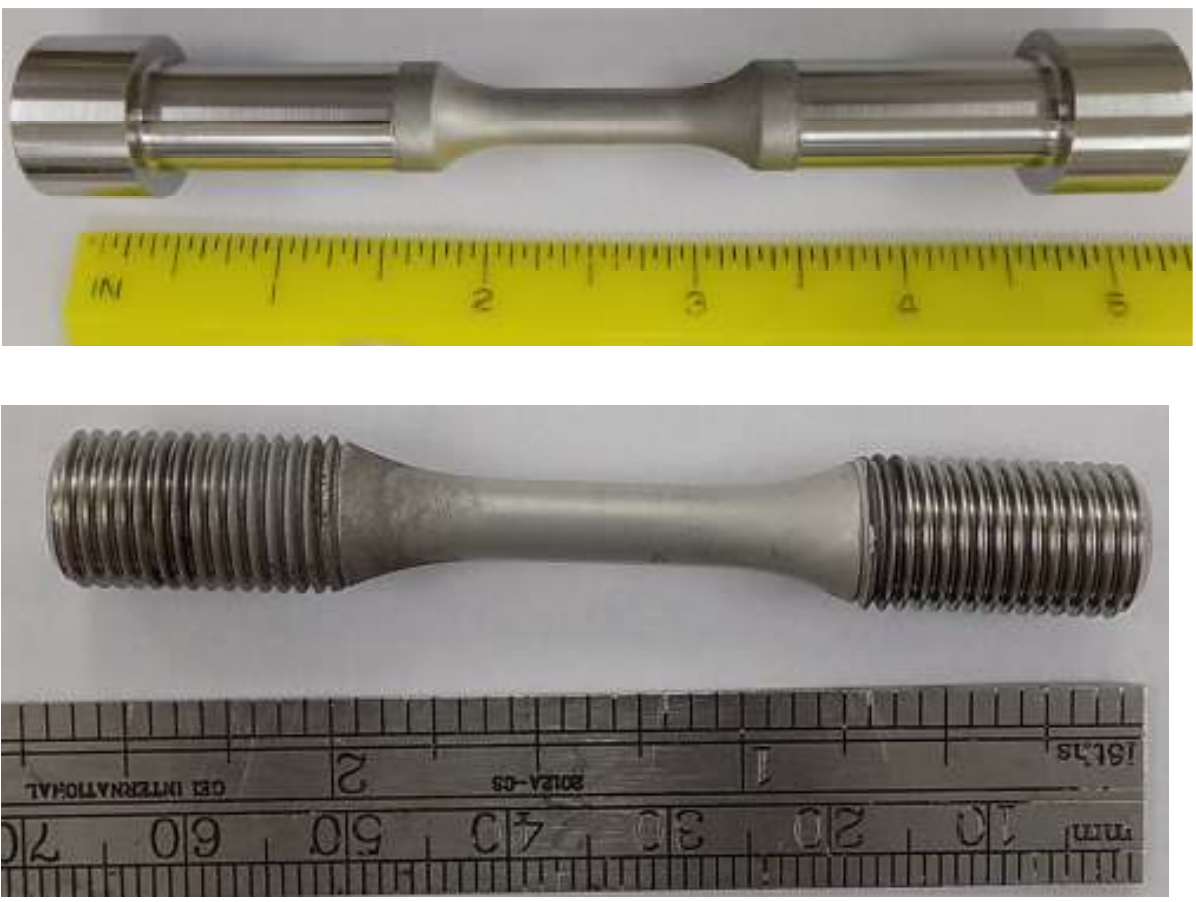

FARADAY תחת TECHNOLOGY, INC. 


\section{Cross Section SEM-EDX}
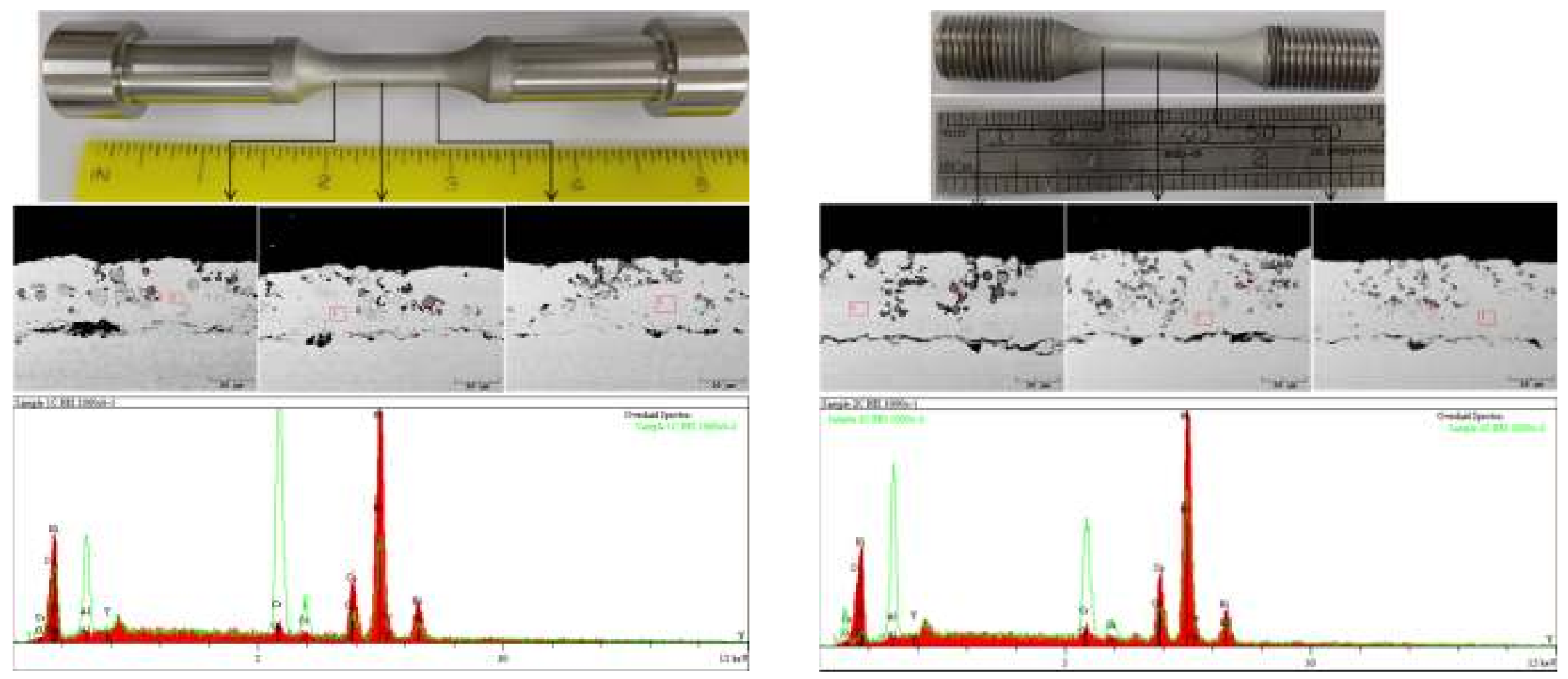

$760^{\circ} \mathrm{C}$ 8h Heat Treatment is not sufficient to fully anneal

FARADAY ЛПЛЛ

TECHNOLOGY, INC. 


\section{Scale up Design}
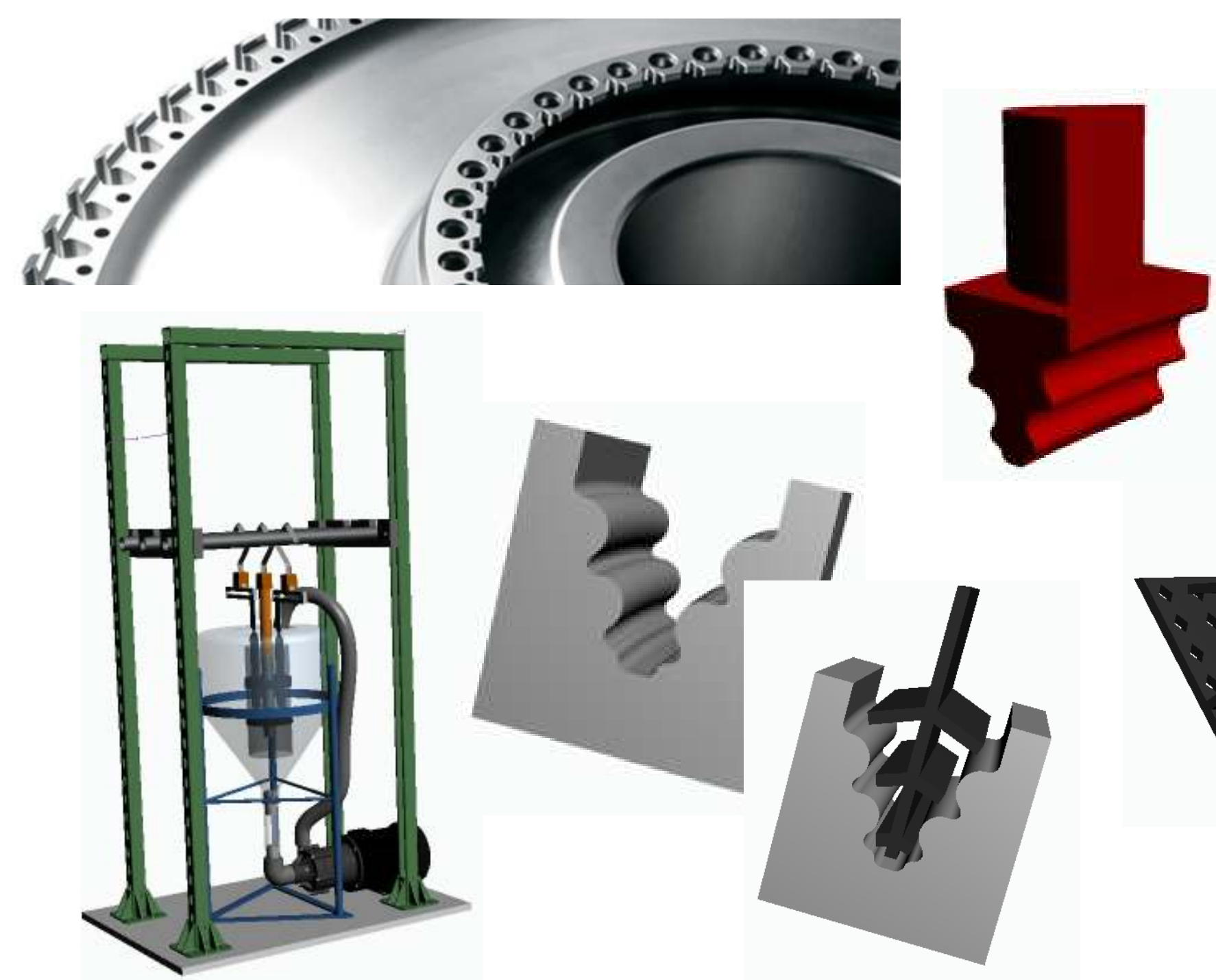

FARADAY תЛП TECHNOLOGY, INC. 


\section{Summary}

- Designed, built, and optimized a scalable electrochemical codeposition system

- Demonstrated the potential to achieve 30 vol\% CrAlY inclusions within the $\mathrm{NiCo}$

- Optimized annealing protocols to dissolve CrAlY particle into NiCo matrix

- Identified potential elemental additions of $\mathrm{Hf}, \mathrm{Si}$, and $\mathrm{Cr}$ could improve hot corrosion resistance

- Demonstrated the potential to improve both hot and accelerated corrosion resistance

- Uniformly coated and annealed conventional low cycle fatigue (LCF) bars

- Designed tooling to enable uniform coating on conventional turbine disk teeth 


\title{
$\longrightarrow$ FARADAY \\ THANK YOU FOR YOUR ATTENTION! QUESTIONS?
}

Financial support: NASA

\author{
Contact Information: \\ Jing Xu, Tim Hall, Maria Inman, or EJ Taylor \\ Phone: 937-836-7749 \\ Email: jenningstaylor@faradaytechnology.com \\ jingxu@faradaytechnology.com \\ timhall@faradaytechnology.com \\ mariainman@faradaytechnology.com
}

